\title{
Zur Frage der Bedeutung des Nervensystems für die Regeneration.
}

\author{
Bemerkung zu der Arbeit S. Morgulis'. \\ Von
}

Prof. Dr. E. Joest in Dresden.

Im 143. Bande dieses Archivs, der mir erst jetzt zu Gesicht kommt, findet sich eine Arbeit von S. Morgulis „Über das Verhältnis des Nervensystems zur Regeneration“. Der genannte Forscher hat in dieser Arbeit seinen eigenen Versuchen eine Zusammenstellung der Literatur über den Gegenstand vorausgeschickt. In Ergänzung dieser Literaturübersicht, die meinen Namen nicht erwähnt, möchte ich hier kurz darauf hinweisen, dass ich bei meinen Transplantationsversuchen an Regenwürmern ${ }^{1}$ ) im Jahre 1897 als erster den Einfluss des Nervensystems auf die Regeneration festgestellt habe.

Es handelte sich bei den für diese Frage in Betracht kommenden Versuchen um die Vereinigung von Teilstücken quer durchtrennter Regenwürmer. Die Vereinigung wurde sowohl mit ungleichnamigen (Kopf- und Schwanzstücken) als auch mit gleichnamigen Teilstücken (z. B. zwei Schwanzstücken) und im ersteren Falle teils in normaler Orientierung der Teilstücke zueinander (Bauch an Bauch, Rücken an Rücken), teils unter verschieden grosser Längsachsendrehung (z. B. Bauch an Rücken) vorgenommen. In allen Fällen wurden viele dauernde Verwachsungen erzielt, die sich bei ungleichnamigen Teilstücken auch auf das Bauchmark erstreckten, sofern die Bauchmarkstümpfe aufeinander passten oder (bei nur geringer Längsachsendrehung) nahe benachbart an der Vereinigungsstelle lagen. Bei der Verbindung gleichnamiger-Teilstücke und bei grösseren Längsachsendrehungen (z. B. Vereinigung von Bauch an Rücken) trat jedoch trotz sonstiger guter Verbindung eine Verwachsung der

1) Transplantationsversuche an Lumbriciden. Arch. f. Entwicklungsmech. Bd. 5 S. 419. 1897. Die Untersuchungen wurden im Institut Prof. Korschelt's in Marburg ausgeführt. 
Bauchmarkstümpfe nicht ein. In diesen Fällen beobachtete ich häufig das Auftreten von ein oder zwei Regeneraten an der Verbindungsstelle, die von dem einen oder von beiden Komponenten der Transplantation ausgingen und die dem dem einzelnen Komponenten jeweils fehlenden Körperende entsprachen. Demgegenüber traten Regenerate an der Vereinigungsstelle bei Transplantationen, die zu einer Verwachsung der Bauchmarke geführt hatten, niemals auf. Aus diesen Tatsachen, wie auch aus dem steten Hervorbrechen der Regeneratknospen an der Ventralseite des Teilstückes, aus dem sie hervorgingen, wie auch endlich aus dem Umstande, dass der Bauchstrang des letzteren sich jeweils kontinuierlich in das zugehörige Regenerat fortsetzte, schloss ich damals, dass die an ihrer Verwachsung verhinderten Nervenketten, indem sie bestrebt sind, die ihnen fehlenden Teile des Systems zu ersetzen, die Regeneration eines Kopfes bzw. eines Schwanzes anregen. Ich fügte hinzu, man könne "das Nervensystem als einen der wichtigsten Faktoren, welche die Regeneration bestimmen, ansehen".

Dieser Anschauung hat sich O. Rabes ${ }^{1}$ ), der in Korschelt's Institut die von mir begonneven Untersuchungen fortsetzte, angeschlossen, und T. H. Morgan ${ }^{2}$ ) hat später (ebenfalls am Regenwurm) durch besondere Versuche bestätigt, dass die Regeneration eines Kopfes in der Tat von dem Vorhandensein des Bauchmarkes abhängig ist.

Auf die weitere Literatur will ich hier nicht eingehen, ich will nur bemerken, dass durch sie die Richtigkeit des von mir seiner Zeit ausgesprochenen Satzes (der natürlich nur für Metazoen gilt) im allgemeinen anerkannt worden ist. Eine Ausuahme macht die Zeit der früben Embryonalentwicklung und anscheinend jene Regeneration im kleinen, wie sie sich bei der einfachen Wundheilung abspielt.

1) Transplantationsversuche an Lumbriciden. Arch. f. Entwicklungsmech. Bd. 13 S. 239.1901.

2) Experimental Studies of the internal Factors of Regeneration in the Earthworm. Arch. f. Entwicklungsmech. Bd. 14 S. 562. 1902. 